\title{
The Effects of the Metacognitive Cue of Fluency on Evaluations about Taste Perception
}

\author{
Antonia Mantonakis ${ }^{1}$, Bryan Galiffi ${ }^{1}$, Ummugulsum Aysan ${ }^{1}$, Randi Beckett ${ }^{2}$ \\ ${ }^{1}$ Department of Marketing, International Business and Strategy, Goodman School of Business, \\ Brock University, St. Catharines, Canada \\ ${ }^{2}$ Department of Psychology, Brock University, St. Catharines, Canada \\ Email: amantonakis@brocku.ca
}

Received December 21 $1^{\text {st }}, 2012$; revised January $22^{\text {nd }}, 2013$; accepted February $21^{\text {st }}, 2013$

\begin{abstract}
The metacognitive cue of fluency is known to affect consumers' evaluations and judgments (Schwarz, 2004). We questioned whether this effect extends to perceived taste experiences, and whether knowledge moderates the effect of fluency on taste evaluations. Across 3 experiments we demonstrate that the metacognitive cue of fluency is used by consumers in evaluating their taste experiences. Whereas disfluent cues are associated with lower taste evaluations for a utilitarian product (Experiment 1), disfluent cues are associated with higher taste evaluations for a hedonic product, especially for knowledgeable consumers (Experiment 2), when compared to a no-label (control) condition. Fluency cues that are intrinsic to the product (e.g., ingredients) however do not have the same effect on judgment about hedonic products (Experiment 3). These findings are important for designing product labels.
\end{abstract}

Keywords: Metacognitive Cues; Fluency; Consumers; Sensory Perceptions

\section{Introduction}

Imagine a consumer is browsing the supermarket, and is offered a taste test of a new orange juice. The consumer can not only taste the juice, but can evaluate the juice based on the look of the packaging, the price, and brand-related information. When consumers evaluate food and beverage products for consumption, the decision to purchase is influenced by many factors such as these. The consumer may rely on product flavor and stated ingredients (intrinsic qualities; Levin \& Gaeth, 1988), or product label design and brand-name information (extrinsic qualities; Leclerc, Schmitt, \& Dube, 1994). Recent research suggests that information extrinsic to a product may actually distort the sensory qualities of a product (Litt \& Shiv, 2012), making the understanding of such extrinsic cues, as well as their effect on product judgment, of upmost importance to consumer psychology researchers. In this paper a cue that might be used by consumers in forming product evaluations is examined: the metacognitive cue of fluency. For instance, how easy or difficult is it to pronounce the name of the orange juice?

Fluency is the subjective ease of attempting a cognitive task, induced by a variety of manipulations produced by many different stimuli (Alter \& Oppenheimer, 2009). There are two types of fluency that are more applicable to product labels than others. Perceptual fluency is the ease of perceiving stimuli arising from visual clarity (e.g., fluent font versus disfluent font; Alter \& Oppenheimer, 2009), and phonological fluency is the ease experienced when pronouncing words, affected by letter combinations (Alter \& Oppenheimer, 2008a, 2008b), syllable transitions (Shah \& Oppenheimer, 2007), and rhyming elements (McGlone \& Tofighbakhsh, 2000). The metacognitive cue of fluency derived from product label information might also serve as a cue to product judgments. But in what ways would these metacognitive cues affect consumers' judgments?
It is theorized that consumers possess naïve theories, which are thoughts and feelings surrounding the meaning of specific fluency experiences, created from memories of past product pairings that produce fluency expectations (Schwarz, 2004). The context specific hypothesis expands on how fluency expectations influence consumers' impressions, moderated by product type (Pocheptsova et al., 2010). The notion is that hedonic products, such as wine or gourmet cheese, are expected to be less familiar and more difficult to recognize. Therefore, hedonic products are preferred when paired with disfluent cues because these cues create the perception that the product is unfamiliar. This perceived unfamiliarity creates the impression of novelty, uniqueness (Song \& Schwarz, 2009), rarity, and exclusivity; desired hedonic product characteristics (Pocheptsova et al., 2010).

The level of fluency preferred across different product categories within the same product type demonstrates the complexity of fluency expectations and the influence of fluency on consumers' judgments (Schwarz, 2004). These fluency expectations are based on a person's naïve theories about the category or product (Schwarz, 2004). To illustrate, participants were shown advertisements regarding everyday cheese or gourmet cheese, either paired with a disfluent perceptual description or a fluent perceptual description. Overall, participants indicated they were more likely to purchase the everyday cheese, verifying the hedonic nature of gourmet cheese. But the results also showed that participants reported higher desirability ratings for the everyday cheese when it was paired with an easyto-read font, and the gourmet cheese when it was paired with a difficult-to-read font (Pocheptsova et al., 2010). These findings support the notion that expected levels of fluency paired with different product types can lead to enhanced desirability. Imbedded within the context specific hypothesis, this study about cheese supports the notion that everyday, or utilitarian products 
are preferred with fluent cues, whereas hedonic products are pre- ferred with disfluent cues (Pocheptsova et al., 2010).

The current research focuses on the effect of fluency on consumers' actual satisfaction of a product during consumption. It is known that taste perception is suggestible and ambiguous (Elder \& Krishna, 2010; Krishna, 2012) and is affected by intrinsic (Levin \& Gaeth, 1988) and extrinsic cues (Leclerc et al., 1994), therefore, the metacognitive cue of fluency may influence consumers' experience of taste.

The current set of experiments makes several key contributions to the sensory, cognitive, and consumer psychology literatures. First, this study examines the effects of the metacognitive cue of fluency on actual taste perception, as opposed to taste ratings (cf. Song \& Schwarz, 2009; Pocheptsova et al., 2010). Second, by examining taste perception, this research examines taste-related evaluations for both fluent and disfluent label conditions in comparison to evaluations of a no-label (control) condition. This type of control has never been employed in examinations of fluency before. Third, in examining taste perception, this research explores the effects of different fluency cues on relatively utilitarian (Experiment 1) versus hedonic (Experiment 2) products. Fourth, by examining taste perception, these studies examine not only the role of fluency cues derived from extrinsic product cues (Experiments 1 and 2), but also intrinsic product cues (Experiment 3). Finally, this research examines the role of product knowledge in the effects of the metacognitive cue of fluency on taste-related evaluations.

\section{Utilitarian and Hedonic Product Pilot Study}

\section{Experimental Design and Procedure}

Twelve participants were recruited from Brock University to participate in this within-subjects pilot study. Using Qualtrics software, participants were presented with a total of four questions in random order. The questions were: "Do you consider orange juice to be a utilitarian product?" "Do you consider wine to be a utilitarian product?" "Do you consider wine to be a hedonic product?" "Do you consider orange juice to be a hedonic product?" Each of the four questions were answered on a 7 point scale $(1=$ Not at all, $7=$ Very much $)$.

\section{Results}

Paired samples t-tests were performed for the set of utilitarian questions, and for the set of hedonic questions. For utilitarian perceptions, participants rated orange juice to be higher $(M=4.83)$ than wine $(M=3.17, t(11)=2.54, p<.03)$. For hedonic perceptions, participants rated wine to be higher $(M=$ $5.58)$ than orange juice $(M=3.50, t(11)=4.61, p<.001)$. Therefore, orange juice was used as the utilitarian product in Experiment 1 and wine was used as the hedonic product in Experiments 2 and 3.

\section{Experiment 1: Perceptual Fluency Derived from Extrinsic Information on Utilitarian Product Evaluations}

The purpose of Experiment 1 was to examine the effect of an extrinsic fluency cue on product judgment. Namely, this experiment varied whether the name of an alleged new orange juice was easy versus difficult to read, and examined participants' evaluation of the taste of the orange juice. The name was presented either before, or after the actual tasting, so as to examine the effect of fluency on actual taste (versus ratings; Lee, Frederick, \& Ariely, 2006). Based on the context specific hypothesis (Pocheptsova et al., 2010), it was predicted that disfluent cues will decrease perceived sensory experiences for utilitarian products.

\section{Experimental Design}

One-hundred-thirty-seven participants (100 women, 37 men) between the ages of $18-62(M=22, S D=5.77)$ were recruited from Brock University and the Niagara community. The study employed a 2 (difficult-to-read font, easy-to-read font) $\times 2$ (label shown before, after) between-subjects design with readability and timing as factors. Readability was manipulated by presenting a fictitious label associated with the product (the grove name, Knollwood) either in easy-to-read black 12 pt Arial (KNOLLWOOD) or difficult-to-read 12 pt Brush Script MT (ZnOLLW000; Song \& Schwarz, 2008) font. The grove name was presented following the sentence "The oranges for this orange juice sample come from the following farm". Timing was manipulated by presenting the grove name either before or after tasting (Lee, Frederick, \& Ariely, 2006; Siegrist \& Cousin, 2009). A control condition where participants did not see any label was also used. Random assignment placed participants to one of these five conditions. Note that the same orange juice was presented in all conditions (unbeknownst to participants).

\section{Procedure}

Participants were informed that they would taste an orange juice sample and then answer some questions about the sample as well as some questions about themselves. Each participant received an unlabeled Styrofoam cup filled with $30 \mathrm{~mL}$ of a private label orange juice. Using Media Lab software, participants were first asked to report their current thirst level, on a 7-point scale $(1=$ Not at all, $7=$ Very thirsty; Winkielman, Berridge, \& Wilbarger, 2005). Participants were asked to report their thirst level because the level of motivation might affect the desire to consume orange juice, thereby altering evaluations.

Participants in the before condition saw the grove name, rated their familiarity with the grove name and then tasted the sample. Familiarity was measured on a 7 -point scale $(1=$ Not at all, $7=$ very familiar). Participants in the after condition first tasted the sample, then saw the grove name and rated their familiarity with the grove name.

After tasting, participants evaluated the sample across four measures. Overall liking was measured by "Overall, how much do you like this orange juice sample?" $(1=$ Not at all, $7=$ Very much; Siegrist \& Cousin, 2009). Willingness-to-buy was measured with the question "How likely is it that you would buy this orange juice?" ( 1 = Not at all, 7 = Very likely; Wszelaki et al., 2005). Willingness-to-pay was measured with participants indicating an amount in Canadian dollars; "How much would you be willing to pay for a $1.89 \mathrm{~L}$ carton of this orange juice?" (Siegrist \& Cousin, 2009). Ability to quench thirst was measured with "How much did this orange juice quench your thirst?" ( 1 = Not at all, 7 = Very much; Winkielman et al., 2005).

After participants tasted and evaluated the orange juice they answered some personal and variety seeking questions. The variety seeking questions were "I like to try different things", "I like a great deal of variety", and "I like new and different 
styles" ( $1=$ Agree, 5 = Disagree; Bruner, 2009). Some of these questions were asked to acquire basic demographic information about the participants whereas others were asked because they might be potential covariates.

\section{Results}

Gender, smoking habits, variety seeking, and familiarity were all used as covariates in a MANOVA. These variables had no effect on the data and will not be discussed further. Thirst was also used as a covariate; it did not have a significant effect on any other dependent variable except ability to quench. The perceived ability to quench thirst was correlated with thirst level $(r=.475, p<.0001)$; a univariate ANOVA with readability and timing as independent variables showed that the difference across the five conditions for the quench measure was more significant when thirst was used as a covariate $(F(4,134)$ $=7.296, p<.0001$ ).

\section{Easy-to-Read Group}

Data were analyzed using a MANOVA with timing and readability as independent variables, and overall liking, willingnessto-buy, willingness-to-pay, and ability to quench thirst as dependent variables. Participants in the easy-to-read before and after conditions gave similar ratings for all the measures when compared to participants in the no-label condition.

\section{Difficult-to-Read Group}

A MANOVA demonstrated that for overall liking, there was a main effect of readability $(F(1,134)=3.62, p<.06)$. Overall liking of the difficult-to-read before group $(M=3.77)$ was significantly lower than the no label group $(M=5.00, t(57)=$ $-3.22, p<.01)$. For willingness-to-buy, the main effect of readability was marginally significant $(F(1,134)=2.89, p$ $<.10$ ), and the interaction between readability and timing was significant $(F(1,134)=4.66, p<.04)$. The willingness-to-buy ratings of the difficult-to-read before group $(M=3.00)$ were significantly lower than the no label group $(M=4.33, t(57)=$ $-2.93, p<.01)$.

For willingness-to-pay, the dollar amounts reported by participants were log-transformed for analysis. The main effect of timing was significant $(F(1,134)=4.60, p<.04)$. The difficult-to-read before group $(M=\$ 2.30, \log M=.32)$ gave significantly lower ratings than the no label group $(M=\$ 2.73, \log M$ $=.42, t(57)=-2.42, p<.02)$. Finally, the difficult-to-read label was also associated with a lowered perceived ability to quench thirst. The difference across the five conditions for the quench measure was marginally significant $(F(4,134)=2.01, p<.10)$. The quench score of the difficult-to-read before condition $(M=$ $3.58)$ was significantly lower than the no label condition $(M=$ $4.67, t(57)=-2.89, p<.01)$.

\section{Discussion}

The results of this experiment provide the first evidence that the metacognitive cue of fluency can alter sensory experiences. When evaluating orange juice, a disfluent name is associated with decreased taste ratings, and diminished willingness-to-buy, willingness-to-pay and perceived ability of the drink to quench thirst. By use of a control condition these results demonstrate that fluency does not produce an advantage rather disfluency creates a disadvantage, at least for a utilitarian product like orange juice. These results also demonstrate that the metacognitive cue of fluency cannot alter product evaluations once the sample has been consumed. Fluency of the label had no effect on ratings after the actual taste experience. This may be because sensory evaluations are not subject to post hoc reevaluations (cf. Lee et al., 2006).

A limitation of Experiment 1 is that it examined only one type of product. In addition, it did not address other potential moderators, such as product knowledge. Do metacognitive fluency cues have the same effect on hedonic product evaluations? Is this relationship moderated by product knowledge?

Prior knowledge about the product being tasted may also play a role in how the fluency cue is interpreted. For example, wine experts are known to focus more on a wine's odor than non-experts (Lawless, 1984), and have more enhanced vocabulary and cognitive schemas, enhancing their ability to discriminate amongst different wine qualities (Lawless, 1984; Hughson \& Boakes, 2001; Ballester, Patris, Symoneaux, \& Valentin, 2008). Further, the amount of prior knowledge is an indicator of prior product exposure (Hughson \& Boakes, 2001), which might suggest that high knowledge consumers have more experience with the product and more developed expectations regarding the level of fluency typically paired with the products' labels. As such, more sophisticated expectations regarding the product being consumed might have a greater influence on the development of a consumer's evaluation of a product when it's being consumed (Whittlesea \& Williams, 1998; 2001; Schwarz, 2004). Taken together, it was predicted that people who are more knowledgeable about a product will be more motivated to evaluate the product accurately (Cardello et al., 1982; Hughson \& Boakes, 2001; Lawless, 1984). This means more knowledgeable consumers might actually be more influenced by fluency cues in some instances.

\section{Linguistic Name Pairs Pilot Study}

\section{Experimental Design and Procedure}

Thirty-two participants were recruited from Brock University. There were three conditions; one condition for winery names, one for grape varietal names, and one for brand names. The three conditions were within subjects, presented to each participant in random order. Each condition included 10 pairs of names. Name pairs consisted of two names that differed in phonological fluency; for an English speaker one name was harder to pronounce than the other name (i.e., Spatzendreck vs. Storybook). Name pairs matched closely on the number of syllables and letters. Using Qualtrics software, a total of 30 name pairs, consisting of 60 individual names were presented in random order, for each condition, for each participant. For each name presented, participants answered five questions in fixed order. Each of the five questions were answered on a 7-point scale $(1=$ Not at all, $7=$ Very much $)$, except for the value measure that had anchors $(1=$ Under $\$ 10,7=$ More than $\$ 35)$. The five questions pertained to perceived fluency, similarity to English, familiarity, value, and prototypically.

\section{Results}

Paired samples $t$-tests were performed for each of the five dependent variables (perceived fluency, similarity to English, familiarity, value, and prototypicality), for each name pair, in 
each condition (winery, grape varietal, and brand). The ideal inclusion criteria for the name pairs were that the two names differed significantly on the fluency and similarity to English measures, but did not differ significantly for the perceived familiarity, value, and prototypically measures.

For the winery condition, nine potential name pairs were examined. ${ }^{1}$ Paired $t$-tests resulted in one pair meeting ideal inclusion criteria. The winery pair Tselepou vs. Titakis was significantly different on the fluency measure $(t(31)=-7.02, p$ $<.0001)$, and the similarity to English measure $(t(31)=-4.25$, $p<.0001)$, but was not significantly different on the perceived familiarity $(t(31)=0.16, p<.89)$, value $(t(31)=1.03, p<.31)$, or prototypically $(t(31)=-0.77, p<.45)$ measures.

For the grape varietal condition, eight potential name pairs were examined. Paired $t$-tests resulted in two pairs meeting ideal inclusion criteria. The grape varietal pair Alvarelhão vs. Alexandrouli was significantly different on the fluency measure $(t(31)=-3.85, p<.0001)$, and the similarity to English measure $(t(31)=-3.73, p<.001)$, but was not significantly different on the perceived familiarity $(t(31)=-0.90, p<.37)$, value $(t(31)$ $=-1.90, p<.07)$, or prototypically $(t(31)=-1.37, p<.18)$ measures. As well, the grape varietal pair Csillam vs. Crato was significantly different on the fluency measure $(t(31)=-8.84, p$ $<.0001)$, and the similarity to English measure $(t(31)=-6.17$, $p<.0001)$, but was not significantly different on the perceived familiarity $(t(31)=-0.70, p<.49)$, value $(t(31)=0.11, p<.91)$, or prototypically $(t(31)=-1.41, p<.17)$ measures.

In summary, this pilot study identified one name pair in the winery condition (Tselepou vs. Titakis), and two name pairs in the grape varietal condition (Alvarelhão vs. Alexandrouli and Csillam vs. Crato). The focus of the next experiments was on only winery names and grape varietal names ${ }^{2}$, as such, the winery pair Tselepou vs. Titakis was used in Experiment 2 and the grape varietal pair Alvarelhão vs. Alexandrouli was used in Experiment 3.

\section{Experiment 2: Linguistic Fluency Derived from Extrinsic Information on Hedonic Product Evaluations}

The purpose of Experiment 2 was to examine the effect of an extrinsic fluency cue (winery name) on the evaluation of a hedonic product (wine). The experiment varied whether the name of the winery associated with a particular wine was easy- versus difficult-to-pronounce and examined participants' evaluations of the wine, including how much they would be willing to pay for the wine. The name was presented before the actual tasting. It was predicted based on the concept of naive theories (Schwarz, 2004), that the level of prior product knowledge will affect participants' fluency expectations resulting in different data patterns for low and high knowledge consumers. Specifically, it was predicted that disfluent names might be perceived to be more rare or unique, especially for more knowledgeable consumers, who may be more motivated, in creating the perception of higher value.

\footnotetext{
${ }^{1}$ Preliminary screening of the raw data showed that most participants, when answering the questionnaire, had missed a question or a few questions. Name pairs with data determined to be missing systematically by the missing variance analyses using SPSS 19 software were removed. The remaining pairs were compensated with estimated means.

${ }^{2}$ The brand name condition of the pilot study was not relevant to Experiments 2 and 3 and therefore will not be discussed further.
}

\section{Experimental Design}

One-hundred-thirty-seven participants (58 women, 79 men) between the ages of $19-62(M=24 S D=7.33)$ who had not already participated in Experiment 1 or the two previously described pilot studies were recruited from Brock University and the Niagara community. Participants were randomly assigned to either the easy-to-pronounce, difficult-to-pronounce, or the no-label (control) condition. The procedure matched that of Experiment 1, with the exception that the timing factor used in Experiment 1 was dropped because the previous experiment showed that fluency only had an effect when experienced before tasting. Participants in the easy-to-pronounce condition read that the wine they are about to taste was from "Titakis Winery", wherein the cue was presented prior to tasting. Participants in the difficult-to-pronounce conditions read that the wine was from "Tselepou Winery", and followed the same sequence variation as the easy-to-pronounce condition. The nolabel group was not presented with either winery name.

\section{Procedure}

The procedure and dependent measures (overall liking, willingness-to-buy, and willingness-to-pay) were similar to Experiment 1 , except participants tasted a $90 \mathrm{ml}$ sample of Chardonnay instead of orange juice, and were provided with a winery name using a $12 \mathrm{pt}$ Times New Roman font, instead of an orange juice grove name, and only before the tasting. Participants were informed of the winery name through text presented on a computer using Media Lab software, "This wine sample has been supplied by Titakis (Tselepou) Winery". The no-label condition was not given any winery information. Note that the same wine was presented in all 3 conditions (unbeknownst to participants). Participants responded to the dependent measures of familiarity, overall liking, willingness-to-buy, and willingness-to-pay. They also indicated their general liking of white wine as well as demographic and variety seeking behaviors (Bruner, 2009). Participants then completed a wine knowledge questionnaire (Hughson \& Boakes, 2001).

\section{Results}

The wine knowledge questionnaire was used to categorize participants as either high or low wine knowledge consumers (Hughson \& Boakes, 2001). Based on this, 92 participants were placed in the high wine knowledge group and 45 participants were placed in the low wine knowledge group. Gender, smoking, and liking of white wine displayed no effects and will not be discussed further.

\section{High Knowledge Participants}

Data were analyzed using t-tests with ease-of-pronouncing as the independent variable, and overall liking, willingness-to-buy, and willingness-to-pay, as dependent variables. Planned independent samples t-tests comparing means for the difficult-topronounce group versus the no-label group for overall liking showed that participants in the difficult-to-pronounce group $(M$ $=4.83)$ indicated they liked the wine more than the no-label group $(M=4.13,(t(58)=-1.72, p<.05,1$-tailed $)$. For willingness-to-buy, participants in the difficult-to-pronounce group $(M$ $=4.43)$ provided higher ratings than the no-label group $(M=$ $3.70, t(58)=-1.78, p<.04,1$-tailed). For willingness to pay, 
participants in the difficult-to-pronounce group $(M=\$ 16.13)$ reported a higher willingness-to-pay than the no-label group $(M$ $=\$ 11.73, t(58)=-2.90, p<.002,1$-tailed).

Planned independent samples t-tests comparing means for the easy-to-pronounce group versus the no-label group for the dependent measures showed no significant differences.

\section{Low Knowledge Participants}

For the low knowledge participants, planned independent samples t-tests comparing means for the difficult-to-pronounce group versus the no-label group, and the easy-to-pronounce group versus the no-label group, on each of the dependent variables (overall liking, willingness-to-buy, and willingness-topay) revealed no significant effects.

\section{Discussion}

This experiment showed that a disfluent extrinsic product cue (i.e., a winery name) enhanced consumers' evaluations when tasting a hedonic product such as wine, which was the case only for more knowledgeable participants. More importantly, higher knowledge participants reported a higher willingness-to-buy and willingness-to-pay for the wine.

Experiment 3 seeks to further establish the boundaries of this knowledge-based fluency effect by examining the effect of ease of processing of ingredient information (derived from a grape varietal name, for instance) on taste-related evaluations. The dependent variables of perceived taste uniqueness and rarity were added to test the supposed underlying perceptions that may be involved in this effect proposed by the context specific hypothesis that explains past findings that have shown a disfluent or difficult-to-read name results in higher novelty ratings (Pocheptsova et al., 2010).

The effect of fluency cues on a consumer's evaluation of a product may also be determined by the source of the fluency. Is it an easy-to-pronounce brand name (extrinsic quality) or an easy-to-pronounce ingredient name (intrinsic quality)? In other words, the type of information the consumer is evaluating, whether extrinsic or intrinsic to the product, may play a role in the consumer's judgment. That is because consumers evaluate intrinsic information differently because it's directly related to ingestion. For instance, food additive names that are difficultto-pronounce are perceived to be more dangerous and hazardous for consumption (Song \& Schwarz, 2009). Experiment 3 investigates this relationship.

\section{Experiment 3: Linguistic Fluency Derived from Intrinsic Information on Hedonic Product Evaluations}

\section{Experimental Design}

One-hundred-twenty-eight participants (86 women, 42 men), between the ages of $19-43(M=22, S D=4.41)$ who had not already participated in Experiments 1 and 2 or the two previously described pilot studies were recruited from Brock University and the Niagara community. Participants were randomly assigned to either the easy-to-pronounce, difficult-to-pronounce, or control condition. For the three levels of linguistic fluency, the researcher presented the participants with either the easy-topronounce red grape varietal name Alexandrouli, the difficultto-pronounce red grape varietal name Alvarelhão, or no grape varietal information, before they sampled a glass of wine. A wine knowledge questionnaire (Hughson \& Boakes, 2001) was administered to participants after they had tasted the wine to determine their pre-experimental wine knowledge, allowing for participants to be classified as either high or low wine knowledge consumers.

\section{Procedure}

The procedure and dependent measures (overall liking, willingness-to-buy, and willingness-to-pay) were the same as Experiment 2, with the exception that participants were presented with a glass of $70 \mathrm{ml}$ of Pinot Noir wine and were provided with only grape varietal information; "you are about to sample a glass of wine made with the grape varietal Alexandrouli (Alvarelhão). Please keep the name Alexandrouli (Alvarelhão) in your mind by repeating it in your head" (Song \& Schwarz, 2009; Pocheptsova et al., 2010). Following these instructions, participants answered questions regarding the dependent measures of overall liking, willingness-to-buy, and willingness-topay with the addition to taste uniqueness and perceived rarity (all on 7-point scales).

\section{Results}

The wine knowledge questionnaire was used to categorize participants as either high or low wine knowledge consumers (Hughson \& Boakes, 2001). Based on this, 64 participants were placed in the high wine knowledge group and 64 participants were placed in the low wine knowledge group. Gender, smoking, liking of red wine, uniqueness, and rarity displayed no effects and will not be discussed further.

\section{High Knowledge Participants}

Planned independent samples t-tests compared means for the easy-to-pronounce group versus the no-label group, and the difficult-to-pronounce group versus the no-label group, on each of the dependent variables (overall liking, willingness-to-buy, and willingness-to-pay). None of these six comparisons revealed significant effects for the high knowledge participants.

\section{Low Knowledge Participants}

Planned independent samples t-tests were carried out, comparing means for the easy-to-pronounce group versus the no-label group. Although there were no differences in means for overall liking for participants in the easy-to-pronounce group $(M=4.62)$ versus the no-label group $(M=4.21), t(43)=.945, p<.18$, 1-tailed), for willingness-to-buy, participants in the easy-to-pronounce group $(M=4.05)$ provided significantly higher ratings than the no-label group $(M=3.13, t(43)=1.95, p<.02$, 1 -tailed $)$. For willingness to pay, participants in the easy-to-pronounce group $(M=\$ 21.29)$ reported a higher willingness-to-pay than the no-label group $(M=\$ 15.56, t(43)=3.02, p<.002$, 1-tailed $)$.

Planned independent samples t-tests comparing means for the difficult-to-pronounce group versus the no-label group for the dependent measures showed no significant differences for the low knowledge participants.

\section{Discussion}

Unlike what was found in Experiment 2 with an extrinsic cue, 
Experiment 3 showed that disfluent cues did not enhance perceived sensory experiences for hedonic products when the cues were derived from intrinsic product information. This could be because intrinsic information is directly related to ingestion and a disfluent cue can indicate a health hazard (Song \& Schwarz, 2009).

Although the ratings differed across conditions with a general preference for the easy-to-pronounce condition, this finding occurred for only consumers with lower knowledge. Perhaps this is the case because low knowledge consumers, given their relative lack of experience with the product, may be even wearier of unfamiliar names, especially when it comes to ingredients (cf. Song \& Schwarz, 2009). This finding contributes to the literature showing that metacognitive cues associated with intrinsic product information are not interpreted by consumers in the same way as cues relating to extrinsic product information.

\section{General Discussion}

The results of three experiments showed that the metacognitive cue of fluency can affect consumers' taste related evaluations, and this is moderated by the type of product (whether utilitarian or hedonic), the type of fluency cue (whether it's about an extrinsic or intrinsic product characteristic), and consumer knowledge. These findings were demonstrated by comparing evaluations associated with different fluency cues in comparison to evaluations made in a control condition. The use of a true control condition was important because it allowed for the identification of data patterns that would not have been found if only evaluating the differences between the fluent versus disfluent conditions.

\section{Theoretical Contributions}

These studies make several key contributions to the fluency, sensory evaluation, and consumer psychology literatures. First, the metacognitive cue of fluency was shown to extend to actual taste-related evaluations as opposed to simple ratings (cf. Song \& Schwarz, 2009; Pocheptsova et al., 2010). This finding confirms that metacognitive fluency cues can affect taste evaluations, when the cues are presented before, not after, the tasting experience (cf. Lee et al, 2006). This was found for utilitarian and hedonic products, showing that a disfluent cue can actually increase ratings for hedonic products (Experiment 2), despite the overwhelming literature stating that only fluent cues enhance product evaluations (Alter \& Oppenheimer, 2009). Second, taste-related evaluations were examined for both fluent and disfluent label conditions in comparison to evaluations for control conditions. By using a true control group, the results showed that at least for utilitarian products (Experiment 1), fluent cues do not necessarily enhance evaluations, rather, it is disfluent cues that lower taste evaluations.

On the other hand, for hedonic products (Experiment 2), it's disfluent cues that enhance evaluations, especially for high knowledge participants. These findings extend past research showing that experts are more sophisticated while evaluating wine because of enhanced vocabulary and motivations (Lawless, 1984; Hughson \& Boakes, 2001; Ballester et al., 2008). Our research shows instead that it may be because higher knowledge consumers are more motivated and persistent; they may simply be more susceptible to using extrinsic cues in in- forming their judgment.

Fourth, in examining taste perception, these experiments showed that fluency cues have a different effect on judgment dependent on the product type (Pocheptsova et al., 2010; Leclerc et al., 1994), as shown in Experiment 1 using a utilitarian product (disfluent cues are associated with lower ratings) and in Experiment 2 with a hedonic product (disfluent cues are associated with higher ratings).

Last, these experiments confirm that fluency cues have a different effect on evaluations depending on whether the information is derived from an extrinsic or intrinsic cue. Disfluent cues enhance ratings for hedonic products when derived from extrinsic information (cf. Leclerc et al., 1994), whereas fluent cues seem to enhance ratings for a hedonic product when derived from intrinsic information (cf. Song \& Schwarz, 2009), this finding was further moderated by prior knowledge. Specifically, lower knowledge participants seemed especially susceptible to fluent intrinsic cues, reporting a higher willingnessto-buy and a higher willingness-to-pay when the ingredient was easier to pronounce. This finding was not the case for higher knowledge consumers.

\section{Managerial Implications}

These studies are important for consideration by marketers when designing product labels. It is important for marketers to consider the product type (utilitarian vs. hedonic) before deciding whether it may be beneficial to use fluency cues as part of the marketing mix, especially when it comes to product packaging. These studies suggest that utilitarian products will be at a disadvantage when presented with disfluent cues whereas hedonic products will be at an advantage when presented with disfluent cues regarding extrinsic product information. It is also important to consider fluency cues in light of other elements of the marketing mix, including price and promotional activities.

\section{Limitations and Future Research}

The first major limitation is that these experiments were conducted in a laboratory setting. This restricted environment is inherently different than a purchasing or consumption scenario and fluency cues would not be considered relevant to product judgments if they were perceived as fabricated (Alter \& Oppenheimer, 2009). Future research should investigate the effects of context and varying experiment locations (e.g., ordering in a restaurant vs. picking up a package from a display shelf in a store), and levels of product prototypicality, on purchase decisions regarding products with varying fluency cues.

Fluency manipulations were reliant on participants' English language competence because participants needed to read and understand the instructions. Therefore, a major limitation for these studies is that participants did not indicate whether English was their first or second language or if they had a known language disorder. Future studies should screen for known language disorders, reading abilities, or English as a second or third language.

Overall, this research is important for understanding sensory perceptions and designing product labels because understanding extrinsic cues used by consumers for evaluating a product's intrinsic characteristics and overall worth has marketing and sensory science implications. Future research should replicate these studies taking the necessary steps to address the identified 
limitations.

\section{Acknowledgements}

We would like to thank Andrew Reynolds from Brock University's Cool Climate Oenology and Viticulture Institute for supplying the wine used in Experiments 2 and 3. We are thankful for the help provided by the Research Assistants at the Consumer Perception and Cognition Laboratory (Matthew Philp \& Lindsey Carey). We would also like to acknowledge the Natural Sciences and Engineering Research Council of Canada, and the Social Sciences and Humanities Research Council of Canada for helping to fund this research.

\section{REFERENCES}

Alter, A. L., \& Oppenheimer, D. M. (2008a). Easy on the mind, easy on the wallet: The role of familiarity and processing fluency in valuation judgments. Psychonomic Bulletin and Review, 15, 985-990. doi:10.3758/PBR.15.5.985

Alter, A. L., \& Oppenheimer, D. M. (2008b). Effects of fluency on psychological distance and mental construal (or why New York is a large city, but New York is a civilized jungle). Psychological Science, 19, 161-167. doi:10.1111/j.1467-9280.2008.02062.x

Alter, A. L., \& Oppenheimer, D. M. (2009). Uniting the tribes of fluency to form a metacognitive nation. Personality and Social Psychology Review, 13, 219-235. doi:10.1177/1088868309341564

Ballester, J., Patris, B., Symoneaux, R., \& Valentin, D. (2008). Conceptual vs. perceptual wine spaces: Does expertise matter. Food Quality and Preference, 19, 267-276. doi:10.1016/j.foodqual.2007.08.001

Bruner, G. (2009). Marketing scales handbook. V5: A compilation of multi-item measures for consumer behavior and advertising research. Chicago, IL: American Marketing Association.

Cardello, A. V., Maller, O., Kapsalis, J. G., Segars, R. A., Sawyer, F. M., Murphy, C., \& Moskowitz, H. R. (1982). Perception of texture by trained and consumer panelists. Journal of Food Science, 47, 11861197. doi:10.1111/j.1365-2621.1982.tb07646.x

Elder, R. S., \& Krishna, A. (2010). The effects of advertising copy on sensory thoughts and perceived taste. Journal of Consumer Research, 36, 748-756. doi:10.1086/605327

Hughson, A., \& Boakes, R. (2001). Perceptual and cognitive aspects of wine expertise. Australian Journal of Psychology, 53, 103-108. doi:10.1080/00049530108255130

Krishna, A. (2012). An integrative review of sensory marketing: Engaging the senses to affect perception, judgment and behavior. Journal of Consumer Psychology, 22, 332-351.

doi:10.1016/j.jcps.2011.08.003

Lawless, H. (1984). Flavour discrimination of white wine by "expert" and non-expert wine consumers. Journal of Food Science, 49, 120123. doi:10.1111/j.1365-2621.1984.tb13686.x

Leclerc, F., Schmitt, B. H., \& Dube, L. (1994). Foreign branding and its effects on product perceptions and attitudes. Journal of Marketing Research, 31, 263-270. doi:10.2307/3152198

Lee, L., Frederick, S., \& Ariely, D. (2006). Try it, you'll like it: The influence of expectation, consumption, and revelation on preferences for beer. Psychological Science, 17, 1054-1058. doi:10.1111/j.1467-9280.2006.01829.x

Levin, I. P., \& Gaeth, G. J. (1988). How consumers are affected by the framing of attribute information before and after consuming the product. Journal of Consumer Research, 15, 374-378. doi: $10.1086 / 209174$

Litt, A., \& Shiv, B. (2012). Manipulating basic taster perception to explore how product information affects experience. Journal of Consumer Psychology, 22, 55-66. doi:10.1016/j.jcps.2011.11.007

McGlone, M. S., \& Tofighbakhsh, J. (2000). Birds of a feather flock conjointly: Rhyme as reason in aphorisms. Psychological Science, 11, 424-428. doi:10.1111/1467-9280.00282

Pocheptsova, A., Labroo, A., \& Dhar, R. (2010). Making products feel special: When metacognitive difficulty enhances evaluation. Journal of Marketing Research, 47, 1059-1069. doi:10.1509/jmkr.47.6.1059

Siegrist, M., \& Cousin, M. (2009). Expectations influence sensory experience in a wine tasting. Appetite, 52, 762-765. doi:10.1016/j.appet.2009.02.002

Schwarz, N. (2004). Metacognitive experiences in consumer judgment and decision making. Journal of Consumer Psychology, 14, 332-348. doi:10.1207/s15327663jep1404_2

Song, H., \& Schwarz, N. (2008). If it's hard to read, it's hard to do: Processing fluency affects effort prediction and motivation. Psychological Science, 19, 986-988. doi:10.1111/j.1467-9280.2008.02189.x

Song, H., \& Schwarz, N. (2009). If it's difficult to pronounce, it must be risky: Fluency, familiarity, and risk perception. Psychological Science, 20, 135-138. doi:10.1111/j.1467-9280.2009.02267.x

Whittlesea, B. W. A., \& Williams, L. D. (1998). Why do strangers feel familiar, but friends don't? The unexpected basis of feelings of familiarity. Acta Psychologica, 98, 141-166. doi:10.1016/S0001-6918(97)00040-1

Whittlesea, B. W. A., \& Williams, L. D. (2001). The discrepancyattribution hypothesis: I. The heuristic basis of feelings of familiarity. Journal of Experimental Psychology: Learning, Memory, and Cognition, 27, 3-13. doi:10.1037//0278-7393.27.1.3

Winkielman, P., Berridge, K., \& Wilbararger, J. (2005). Unconscious affective reactions to masked happy versus angry faces influence consumption behavior and judgments of value. Personality and Social Psychology Bulletin, 31, 121-135. doi:10.1177/0146167204271309

Wszelaki, D., Walker, S., Liggett, R., Miller, S., \& Kleinhenz, M. (2005). Consumer liking and descriptive analysis of six varieties of organically grown edamame-type soybean. Food Quality and Preference, 16, 651-658. doi:10.1016/j.foodqual.2005.02.001 